\title{
Preface to BIT 52:1
}

\author{
Axel Ruhe
}

Published online: 22 February 2012

(C) Springer Science + Business Media B.V. 2012

With this issue, we start the fifty second year of BIT and the tenth volume that I have the pleasure and honor to collect and edit. We hope that our content gives you an interesting reflection of what is going on in the BIT area of the scientific field and that you, our readers, find some nonfinished open ends to follow and continue the work on our never finished scientific anthill.

Let me take this opportunity to say thank you to Erkki Somersalo, that now leaves the BIT editorial board. We wish him good luck in his further endeavors and are searching a replacement to cover his area.

Let us look at the contents of this issue! These papers have been sent in to us and found to be ready for publication now:

Winfried Auzinger and Magdalena Łapińska investigate the convergence behavior of rational multistep methods for stiff systems of ordinary differential equations. A rational Adams method is regarded as a modification of an exponential Adams method, and then it is shown how the theory of exponential integrators can be applied. Special interest is given semilinear equations, where the stiff linear part is dissipative and the nonlinear part is Lipschitz continuous.

Romina Gobbi and Renato Spigler use wavelets to solve a singularly perturbed elliptic problem. They compare a Shannon collocation, based on sinc functions, to the use of autocorrelation functions of the kind that is usual in signal processing. The aim is to find out how wavelets can be used to solve differential equations with some of the success that they have shown in signal processing.

Martin Hanke, Lauri Harhanen, Nuutti Hyvönen, and Eva Schweickert study the inverse problem of finding a convex source, that gives a prescribed boundary potential

\footnotetext{
A. Ruhe (凶)

School of Computer Science and Communications (CSC), Royal Institute of Technology (KTH), 10044 Stockholm, Sweden

e-mail: ruhe@kth.se
} 
of a Poisson equation over a bounded 3 dimensional domain. A test on an electrical impedance tomography application is reported.

Antti Hannukainen studies preconditioned iterations to solve a mixed finite element approximation of a Poisson problem. It gives rise to an indefinite matrix of saddle point type. He uses preconditioners, designed for continuous piecewise linear finite element discretizations of the Laplace operator, as preconditioners for this mixed problem. Convergence is established when the mesh size tends to zero.

Mihály Kovács, Stig Larsson, and Fredrik Lindgren analyze the weak error of semidiscrete finite element methods for linear stochastic partial differential equations, driven by additive noise. They derive an error representation formula and apply it to several stochastic evolution equations. The rate of weak convergence appears here to be twice the rate of strong convergence, up to a logarithmic factor.

Raphael Kruse studies bistability of multistep methods for stochastic differential equations. A two sided error estimate is used to establish strong convergence of several multistep methods.

Grigory Milstein and Michael Tretyakov describe a layer method that uses a probabilistic representation of the Navier Stokes equations. It is an alternative to the traditional finite elements, spectral and finite difference methods.

Allan Antti Antero Perämäki is interested in a reconstruction algorithm in impedance tomography using a R-linear Beltrami equation. Convergence of different discretizations are compared and it is found that a polar representation is to be preferred.

Evgenija D. Popova describes the solution sets of a parameter dependent linear system of equations. The parameters vary inside prescribed intervals. A special study is made of two dimensional systems depending on several parameters.

Siegfried M. Rump gives new bounds on the errors of floating point summation and dot product that improve the standard Wilkinson bounds. They use a new tool for error estimation which gives a shorter proof under fewer restrictions.

Driss Sbibih, A. Serghini, and A. Tijini consider splines in 3 variables. A local basis is used for quasi interpolation that is exact for polynomials up to a certain degree, and can be computed without solving a linear system. Convergence for refined regular tetrahedral partitions of the region is demonstrated on a numerical example.

Liping Wen and Xiong Liu study nonlinear neutral delay differential equations. They give conditions for the stability of the equation itself, as well as for the solution by an A-stable one leg time stepping method.

I wish you all an interesting read!

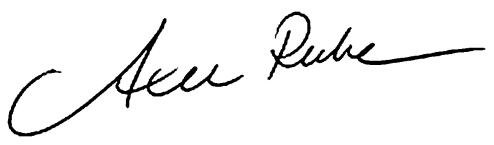

Axel Ruhe 